\title{
WECC Composite Load Model Parameter Identification Using Deep Learning Approach
}

This paper was downloaded from TechRxiv (https://www.techrxiv.org).

\section{LICENSE}

CC BY 4.0

SUBMISSION DATE / POSTED DATE

$21-01-2022$ / 27-01-2022

CITATION

khazeiynasab, Seyyed Rashid (2022): WECC Composite Load Model Parameter Identification Using Deep Learning Approach. TechRxiv. Preprint. https://doi.org/10.36227/techrxiv.18843125.v1

DOI

10.36227/techrxiv.18843125.v1 


\section{WECC Composite Load Model Parameter Identification Using Deep Learning Approach}

\author{
Seyyed Rashid Khazeiynasab \\ Electrical and Computer Engineering \\ University of Central Florida \\ rashid@knights.ucf.edu
}

\author{
Junbo Zhao \\ Electrical and Computer Engineering \\ University of Connecticut \\ junbo@uconn.edu
}

\author{
Nan Duan \\ Lawrence Livermore National Laboratory \\ duan4@llnl.gov
}

\begin{abstract}
The increased integration of distributed energy resources (DERs) has significantly changed the load composition. As compared to traditional static or induction motor loads, these inverter-based resources (IBRs) exhibit different behavior. Then, conventional dynamic load modeling with ZIP and induction motors $(\mathrm{ZIP}+\mathrm{IM})$ is no longer adequate to address the current load characteristic transitions. Western Electricity Coordinating Council (WECC) has developed the composite load model with distributed generation (CMPLDWG) to accurately represent static load, induction motors, and IBRs. In this paper, we propose a novel deep learning-based method using a conditional variational autoencoder (CVAE) to estimate the CMPLDWG model's posterior distributions. The effectiveness of the proposed method is validated using an IEEE 39-bus test system. The results show that the proposed approach can accurately and efficiently estimate the parameters' posterior distributions.

Index Terms-Conditional variational autoencoders, deep learning, WECC composite load model, parameter identification.
\end{abstract}

\section{INTRODUCTION}

Dynamic load modeling is crucial for conducting stability analysis and power system simulations. Load model parameter identification is essential to power systems studies, such as conservation voltage reduction, voltage stability assessment, and power system dynamics [1]-[4]. The identification of parameters remains a challenge for academic researchers and industrial practitioners due to the rising diversity of loads and the integration of distributed energy resources (DERs). Many parameter identification approaches use measurementbased approaches by taking voltage and power measurements during fault-induced delayed-voltage-recovery (FIDVR) events to determine the parameters of dynamic load models [5], [6].

One of the developed models for load is the ZIP model that contains an algebraic constant impedance component $(\mathrm{Z})$, constant current component (I), and constant power component (P). The ZIP and induction motor (ZIP+IM) models have been widely used in industry and academia for decades to quantify load characteristics in composite load models [7]. Several approaches have been proposed to estimate the parameters of the ZIP+IM model [8]-[10]. A multimodal Long Short Term Memory (LSTM) deep learning approach is employed in [8] to identify the composite load model's time-varying parameters. In [9], a deep generative based on the LSTM network is presented for probabilistic time-varying parameter identification. The method in [10] proposes an imitation and transfer q-learning-based method.
In recent years, the composition of loads has changed significantly as more DERs have been integrated into distribution systems. Therefore, loads' dynamic behavior is difficult to predict using traditional static and dynamic load models, especially for FIDVR events. Thus, WECC has developed the composite load model with distributed generation (CMPLDWG). The proposed model includes static load, different threephase induction motors, single-phase $\mathrm{A} / \mathrm{C}$ motors, electronic load, and DERs to accurately describe modern energy grids characteristics. In [11], the parameters of a dynamic composite load model were obtained by using matrix decomposition and clustering techniques. However, the model does not include the newly approved aggregated DER. There are two steps in the identification process in [12]: a deep q-learning network is used to identify a load composition that approximates transient dynamics, and then Monte Carlo simulations are used to determine the best fit parameter set. However, it is very sensitive to hyperparameters and requires a lot of tuning for convergence. Moreover, its implementation is also complicated since a lot of effort is needed to stabilize deep q-learning. The evolutionary deep reinforcement learning approach proposed in [5] has good performance, but the computational cost prevents its online application.

In this paper, we propose to develop a conditional variational autoencoder (CVAE) method for parameter identification to overcome the challenges caused by the nonlinearity of the CMPLDWG model. Using an encoding-decoding architecture, CVAE describes a set of nonlinear latent variables to summarize the probability distribution function (PDF) of the model parameters. Using Artificial Neural Networks (ANNs), the encoder estimates latent variables' PDF based on the measurements. With a Rectified Linear Unit (ReLU), the decoder converts the latent variables PDFs into actual model parameters PDFs. The advantages of the proposed approach are 1) The computational efficiency is high, and only a few samples are needed; 2) The proposed method uses simulation and does not require a state-space model or likelihood function for the dynamic load model. Commercial software already contains the load model, making implementation much easier and enabling use in real-world applications. 3) As compared to existing reinforcement and deep learning techniques, this method is easier to implement.

The rest of this paper is organized as follows. Section II introduces the CMPLDWG model and discusses the load 
components and the associated parameters. Section III shows the problem statement and introduces the proposed method. Section IV shows the numerical results and finally the conclusion is drawn in Section $\mathrm{V}$.

\section{WECC LOAD MODEL}

This paper considers the comprehensive WECC composite load model, which consists of three parts: feeder, substation, and load as shown in Fig. 1. The substation section is composed of a transformer and a shunt capacitor. The feeder section is represented by an equivalent feeder model. The load section consists of three induction motors with different dynamic characteristics, i.e., a single-phase induction motor, a static load, an electronic load, and a distributed generator. Three three-phase motors represent three distinct types of dynamic components. Motor A represents three-phase induction motors with low inertia driving constant torque loads, such as an air conditioning compressor. Motor B represents three-phase induction motors with high inertia driving variable torque loads such as air handling systems. Motor $\mathrm{C}$ represents three-phase induction motors with low inertia driving variable torque loads such as the common centrifugal pumps [13]. The details of each type of load are discussed in the following.

\section{A. Three-Phase Induction Motors}

Three of the four motors in Fig. 1 are three-phase induction motors. The following differential and algebraic equations represent the dynamical model of these motors [12], [14]:

$$
\begin{aligned}
& \frac{d E_{\mathrm{q}}^{\prime}}{d t}=-\frac{1}{T_{\mathrm{po}}}\left[E_{\mathrm{q}}^{\prime}+\left(L_{\mathrm{s}}-L_{\mathrm{p}}\right) i_{\mathrm{d}}\right]-\omega_{0} s E_{\mathrm{d}}^{\prime}, \\
& \frac{d E_{\mathrm{d}}^{\prime}}{d t}=-\frac{1}{T_{\mathrm{po}}}\left[E_{\mathrm{d}}^{\prime}-\left(L_{\mathrm{s}}-L_{\mathrm{p}}\right) i_{\mathrm{q}}\right]+\omega_{0} s E_{\mathrm{q}}^{\prime}, \\
& \frac{d E_{\mathrm{q}}^{\prime \prime}}{d t}=\frac{1}{T_{\mathrm{ppo}}}\left[E_{\mathrm{q}}^{\prime}-E_{\mathrm{q}}^{\prime \prime}+i_{\mathrm{d}}\left(L_{\mathrm{p}}-L_{\mathrm{pp}}\right)\right]-\frac{1}{T_{\mathrm{po}}}\left[E_{\mathrm{q}}^{\prime}+i_{\mathrm{d}}\left(L_{\mathrm{s}}-L_{\mathrm{p}}\right)\right] \\
& +\left(1-T_{\mathrm{po}}\right) E_{\mathrm{d}}^{\prime}-\omega_{0} s E_{\mathrm{d}}^{\prime \prime}+s \omega_{0}, \\
& \frac{d E_{\mathrm{d}}^{\prime \prime}}{d t}=\frac{1}{T_{\mathrm{ppo}}}\left[E_{\mathrm{d}}^{\prime}-E_{\mathrm{d}}^{\prime \prime}+i_{\mathrm{q}}\left(L_{\mathrm{p}}-L_{\mathrm{pp}}\right)\right]-\frac{1}{T_{\mathrm{po}}}\left[E_{\mathrm{d}}^{\prime}+i_{\mathrm{q}}\left(L_{\mathrm{s}}-L_{\mathrm{p}}\right)\right] \\
& +\left(1-T_{\mathrm{po}}\right) E_{\mathrm{q}}^{\prime}+\omega_{0} s E_{\mathrm{q}}^{\prime \prime}-s \omega_{0}, \\
& \frac{d s}{d t}=-\frac{1}{2 H}\left[E_{\mathrm{d}}^{\prime \prime} i_{\mathrm{d}}+E_{\mathrm{q}}^{\prime \prime} i_{\mathrm{q}}-T_{\mathrm{m}} \omega_{0} E_{\mathrm{trq}}\right], \\
& i_{\mathrm{d}}=\frac{1}{R_{\mathrm{a}}^{2}+L_{\mathrm{pp}}^{2}}\left[R_{\mathrm{a}}\left(V_{\mathrm{d}}-E_{\mathrm{d}}^{\prime \prime}\right)+L_{\mathrm{pp}}\left(V_{\mathrm{q}}-E_{\mathrm{q}}^{\prime \prime}\right)\right] \\
& i_{\mathrm{q}}=\frac{1}{R_{\mathrm{a}}^{2}+L_{\mathrm{pp}}^{2}}\left[R_{\mathrm{a}}\left(V_{\mathrm{q}}-E_{\mathrm{q}}^{\prime \prime}\right)-L_{\mathrm{pp}}\left(V_{\mathrm{d}}-E_{\mathrm{d}}^{\prime \prime}\right)\right] \\
& p_{3 \emptyset}=\frac{R_{\mathrm{a}}\left(V_{\mathrm{d}}^{2}+V_{\mathrm{q}}^{2}-V_{\mathrm{d}} E_{\mathrm{d}}^{\prime \prime}-V_{\mathrm{q}} E_{\mathrm{q}}^{\prime \prime}\right)-L_{\mathrm{pp}}\left(V_{\mathrm{d}} E_{\mathrm{q}}^{\prime \prime}-V_{\mathrm{q}} E_{\mathrm{d}}^{\prime \prime}\right)}{R_{\mathrm{a}}^{2}+L_{\mathrm{pp}}^{2}} \\
& q_{3 \emptyset}=\frac{L_{\mathrm{pp}}\left(V_{\mathrm{d}}^{2}+V_{\mathrm{q}}^{2}-V_{\mathrm{d}} E_{\mathrm{d}}^{\prime \prime}-V_{\mathrm{q}} E_{\mathrm{q}}^{\prime \prime}\right)-R_{\mathrm{a}}\left(V_{\mathrm{d}} E_{\mathrm{q}}^{\prime \prime}-V_{\mathrm{q}} E_{\mathrm{d}}^{\prime \prime}\right)}{R^{2}} .
\end{aligned}
$$

where $H$ is the inertia constant, $R_{\mathrm{a}}$ is the stator resistance, $E_{\mathrm{d}}^{\prime}$, $E_{\mathrm{q}}^{\prime}$ are the d-axis and q-axis transient voltages, $E_{\mathrm{d}}^{\prime \prime}, E_{\mathrm{q}}^{\prime \prime}$ are the d-axis and q-axis sub-transient voltages, $T_{\mathrm{po}}$, and $T_{\mathrm{ppo}}$ are transient and sub-transient open-circuit time constant. $L_{\mathrm{s}}, L_{\mathrm{p}}$, and $L_{\mathrm{pp}}$ are the synchronous reactance, transient reactance, and sub-transient reactance.

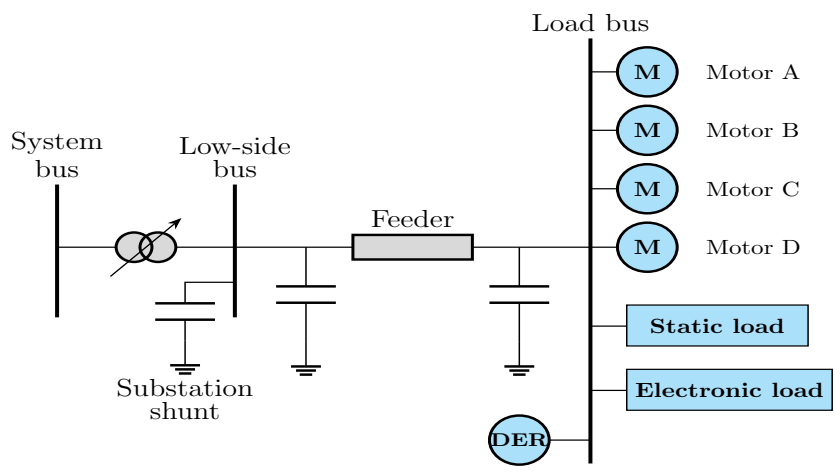

Fig. 1. The structure of the WECC composite load model.

\section{B. Static Load Model}

In CMPLDWG, the static load is represented by the ZIP model. The corresponding active and reactive power are as follows [14]:

$Q_{\text {zip }}=Q_{0}\left[Q_{1 \mathrm{c}}\left(\frac{V}{V_{\mathrm{o}}}\right)^{Q_{1 \mathrm{e}}}+Q_{2 \mathrm{c}}\left(\frac{V}{V_{\mathrm{o}}}\right)^{Q_{2 \mathrm{e}}}+Q_{\mathrm{z}, 3}\right]\left(1+Q_{\mathrm{f}} \Delta f\right)$,

$P_{\text {zip }}=P_{0}\left[P_{1 \mathrm{c}}\left(\frac{V}{V_{\mathrm{o}}}\right)^{P_{1 \mathrm{e}}}+P_{2 \mathrm{c}}\left(\frac{V}{V_{\mathrm{o}}}\right)^{P_{2 \mathrm{e}}}+P_{\mathrm{z}, 3}\right]\left(1+P_{\mathrm{f}} \Delta f\right)$,

$P_{0}=P_{\text {load }}\left(1-F_{\mathrm{MA}}-F_{\mathrm{MB}}-F_{\mathrm{MC}}-F_{\mathrm{MD}}-F_{\mathrm{el}}\right)$,

$Q_{0}=P_{0} \tan \left(\cos ^{-1}(\mathrm{p} . \mathrm{f})\right)$,

where $P_{1 \mathrm{c}}$ and $Q_{1 \mathrm{c}}$ are fractions of constant current load, $P_{2 \mathrm{c}}$ and $Q_{2 \mathrm{c}}$ are percentages of constant current impedance, $P_{\mathrm{f}}$ and $Q_{\mathrm{f}}$ are fractions of static load that is frequency dependent, and $\Delta f$ is frequency dependency factor [7]. All of the $P_{1 \mathrm{c}}, P_{2 \mathrm{c}}, Q_{1 \mathrm{c}}$, and $Q_{2 \mathrm{c}}$ are positive and smaller than 1 [12].

\section{Electronic Load}

The electronic load is intended to model all consumer loads that are connected to the system via an inverter-based interface. Under certain voltage ranges and at specified voltage levels, active and reactive power consumption is set to a constants level and linearly decrease as the voltage drops below the specified voltage range to represent the under-voltage trip of the device. The active and reactive power consumption of the electronic load are defined as follows:

$$
\begin{array}{ll}
F_{\mathrm{vl}}= \begin{cases}1, & \text { if } V \geq V_{\mathrm{d} 1} \\
\frac{V-V_{\mathrm{d} 2}}{V_{\mathrm{d} 1}-V_{\mathrm{d} 2}} & \text { if } V_{\mathrm{min}} \leq V<V_{\mathrm{d} 1} \\
0, & \text { if } V<V_{\mathrm{d} 2} \\
\frac{V_{\mathrm{min}}-V_{\mathrm{d} 2}+F_{\mathrm{rcel}}\left(V-V_{\min }\right)}{V_{\mathrm{d} 1}-V_{\mathrm{d} 2}}, & \text { if } V_{\mathrm{d} 2} \leq V<V_{\mathrm{d} 1}\end{cases} \\
P_{\text {elc }}=F_{\mathrm{vl}} P_{\mathrm{elc}, 0}, \\
Q_{\text {elc }}=\tan \left(\cos ^{-1}\left(\mathrm{p} . \mathrm{f}_{\mathrm{elc}}\right)\right) P_{\mathrm{elc}},
\end{array}
$$

where $P_{\text {elc }, 0}$ is initial power of electronic load, p.f $f_{\text {elc }}$ is the power factor of electronic load, $F_{\text {rcel }}$ is the fraction of 
electronic load that can be restarted after a fault is cleared, $V_{\text {min }}$ tracks the minimum bus voltage during the transient, $V_{\mathrm{d} 1}$ is the voltage threshold at which the electronic load starts to trip, $V_{\mathrm{d} 2}$ indicates the voltage threshold at which all the electronic load trips [14].

\section{Single-phase Induction Motor}

The single-phase induction motor can model the protective devices and the compressors. Using exponential functions, the motor's real and reactive power are divided into three states based on the bus voltage, $V_{\mathrm{t}}$, stall voltage, $V_{\text {stall }}$, and compressor bread down voltage $V_{\text {brk }}$. The active and reactive power consumed by a single-phase motor during the different states are as follows [12], [14].

$P_{\mathrm{sm}}= \begin{cases}P_{\mathrm{sm}, 0}+K_{\mathrm{p} 1}\left(V_{\mathrm{t}}-V_{\mathrm{brk}}\right)^{N_{\mathrm{p} 1},}, & \text { if } V_{\mathrm{t}}>V_{\mathrm{brk}} \\ P_{\mathrm{sm}, 0}+K_{\mathrm{p} 2}\left(V_{\mathrm{brk}}-V_{\mathrm{t}}\right)^{N_{\mathrm{p} 2},}, & \text { if } V_{\mathrm{stall}}<V_{\mathrm{t}} \leq V_{\mathrm{brk}} \\ \frac{V_{\mathrm{t}}^{2}}{R_{\mathrm{stal1}}}, & \text { if } V_{\mathrm{stall}} \geq V_{\mathrm{t}}\end{cases}$
$Q_{\mathrm{sm}}= \begin{cases}Q_{\mathrm{sm}, 0}+K_{\mathrm{q} 1}\left(V_{\mathrm{t}}-V_{\mathrm{brk}}\right)^{N_{\mathrm{q} 1},} & \text { if } V_{\mathrm{t}}>V_{\mathrm{brk}} \\ Q_{\mathrm{sm}, 0}+K_{\mathrm{q} 2}\left(V_{\mathrm{brk}}-V_{\mathrm{t}}\right)^{N_{\mathrm{q} 2},}, & \text { if } V_{\text {stall }}<V_{\mathrm{t}} \leq V_{\mathrm{brk}} \\ \frac{-V_{\mathrm{t}}^{2}}{X_{\text {stall }}}, & \text { if } V_{\text {stall }} \geq V_{\mathrm{t}}\end{cases}$

\section{Proposed CVAE Method}

Assuming the prior distribution for parameter $\boldsymbol{x}$ as $\pi(\boldsymbol{x})$. Bayes's theorem allows us to write its posterior distribution $p(\boldsymbol{x} \mid \boldsymbol{y})$ in terms of the prior distribution and the observation distribution $p(\boldsymbol{y} \mid \boldsymbol{x})$ [6]. The form of this posterior is

$$
p(\boldsymbol{x} \mid \boldsymbol{y}) \propto f(\boldsymbol{y} \mid \boldsymbol{x}) \pi(\boldsymbol{x}),
$$

where $f(\boldsymbol{y} \mid \boldsymbol{x})$ is the data likelihood, which describes the observation process. Explicit likelihood functions are computationally costly for the parameter calibration problem. A variational approach is used in this paper in order to approximate the posterior distribution of (1) for a new measurement which is denoted by $p_{\text {ap }}(\boldsymbol{x} \mid \boldsymbol{y})$.

\section{A. CVAE for Parameter Identification}

Variational autoencoders (VAE) are directed generative models with latent variables [15]. In the VAE, a latent variable $\boldsymbol{z}$ is generated from the prior distribution $\pi(\boldsymbol{x})$, and then the data $x$ are generated from the generative distribution $p_{\boldsymbol{\alpha}_{\text {vae }}}(\boldsymbol{x} \mid \boldsymbol{z})$, which is modeled by a neural network with parameters $\alpha_{\text {vae }}$. Back propagation and stochastic gradient descent can be used to optimize a variational lower bound, and $\alpha_{\text {vae }}$ is tuned by maximizing the likelihood of the training data points [16]. However, unlike CVAE models, we have no control over the VAE data generation process.

We use CVAE to represent the CMPLDWG nonlinear relationship. The CVAE consists of three modules: an encoder, a latent space, and a decoder. The encoder with parameter $\alpha^{\prime}$ gets the input $\boldsymbol{y}$ and turns it into a Gaussian distribution $p_{\alpha^{\prime}}(\boldsymbol{z} \mid \boldsymbol{y})$. CVAE first transforms the inputs into a lower dimensional space $z$, called the latent space [17]. The decoder, parameterized with $\bar{\alpha}$ uses $\boldsymbol{y}$ and samples from latent space to produce $p_{\bar{\alpha}}(\boldsymbol{x} \mid \boldsymbol{y}, \boldsymbol{z})$. The distribution of $p(\boldsymbol{x} \mid \boldsymbol{y})$ is given as follow

$$
p(\boldsymbol{x} \mid \boldsymbol{y}) \approx p_{\text {ap }}(\boldsymbol{x} \mid \boldsymbol{y})=\int_{\boldsymbol{z}} p_{\alpha^{\prime}}(\boldsymbol{z} \mid \boldsymbol{y}) p_{\bar{\alpha}}(\boldsymbol{x} \mid \boldsymbol{y}, \boldsymbol{z}) d \boldsymbol{z} .
$$

The network can not be trained directly to only produce $\boldsymbol{x}$ [18]. To overcome this problem, we use a recognition network $\kappa_{\tilde{\alpha}}(\boldsymbol{z} \mid \boldsymbol{y}, \boldsymbol{x})$, modelled by an additional neural network and governed by the trainable network parameters $\tilde{\alpha}$, which is a proposal distribution. This function can help select values of $\boldsymbol{z}$, which are likely producing $\boldsymbol{x}$. Then (2) can be written as follows:

$$
\begin{aligned}
p_{\mathrm{ap}}(\boldsymbol{x} \mid \boldsymbol{y})= & \int_{\boldsymbol{z}} \frac{p_{\alpha^{\prime}}(\boldsymbol{z} \mid \boldsymbol{y}) p_{\bar{\alpha}}(\boldsymbol{x} \mid \boldsymbol{y}, \boldsymbol{z})}{\kappa_{\tilde{\alpha}}(\boldsymbol{z} \mid \boldsymbol{y}, \boldsymbol{x})} \kappa_{\tilde{\alpha}}(\boldsymbol{z} \mid \boldsymbol{y}, \boldsymbol{x}) d \boldsymbol{z} \\
& =\mathbb{E}_{\kappa_{\tilde{\alpha}}(\boldsymbol{z} \mid \boldsymbol{y}, \boldsymbol{x})}\left(\frac{p_{\alpha^{\prime}}(\boldsymbol{z} \mid \boldsymbol{y}) p_{\bar{\alpha}}(\boldsymbol{x} \mid \boldsymbol{y}, \boldsymbol{z})}{\kappa_{\tilde{\alpha}}(\boldsymbol{z} \mid \boldsymbol{y}, \boldsymbol{x})}\right) .
\end{aligned}
$$

The goal is to find the parameters of the network to maximize the log-likelihood of $p_{\text {ap }}(\boldsymbol{x} \mid \boldsymbol{y})$ over the dataset, i.e

$$
\begin{aligned}
& \underset{\boldsymbol{\Theta}_{\text {cvae }}}{\operatorname{argmax}} \log p_{\text {ap }}(\boldsymbol{x} \mid \boldsymbol{y})= \\
& \underset{\boldsymbol{\Theta}_{\text {cvae }}}{\operatorname{argmax}} \log \mathbb{E}_{\kappa_{\tilde{\alpha}}(\boldsymbol{z} \mid \boldsymbol{y}, \boldsymbol{x})}\left(\frac{p_{\alpha^{\prime}}(\boldsymbol{z} \mid \boldsymbol{y}) p_{\bar{\alpha}}(\boldsymbol{x} \mid \boldsymbol{y}, \boldsymbol{z})}{\kappa_{\tilde{\alpha}}(\boldsymbol{z} \mid \boldsymbol{y}, \boldsymbol{x})}\right) .
\end{aligned}
$$

where $\boldsymbol{\Theta}_{\text {cvae }}$ is the CVAE model parameter, which is $\boldsymbol{\Theta}_{\text {cvae }}=$ $\alpha^{\prime} \cup \bar{\alpha} \cup \tilde{\alpha}$. By using Jensen's inequality, the evidence lower bound (ELBO) is given as

$$
\begin{aligned}
& \log p_{\mathrm{ap}}(\boldsymbol{x} \mid \boldsymbol{y})= \\
& -\mathbb{E}_{\kappa_{\tilde{\alpha}}(\boldsymbol{z} \mid \boldsymbol{y}, \boldsymbol{x})}\left[p_{\bar{\alpha}}(\boldsymbol{x} \mid \boldsymbol{y}, \boldsymbol{z})\right]+\mathfrak{C}\left(\kappa_{\tilde{\alpha}}(\boldsymbol{z} \mid \boldsymbol{y}, \boldsymbol{x}) \| p_{\alpha^{\prime}}(\boldsymbol{z} \mid \boldsymbol{y})\right) \geq \text { ELBO, }
\end{aligned}
$$

where $\mathfrak{C}(\cdot \| \cdot)$ is the Kullback-Leibler divergence between two distributions and can not be negative. Now, the ELBO can be optimized via stochastic gradient descent algorithm [19], [20]. Fig. 2 illustrates the graphical model of a CVAE during the training and test phases.

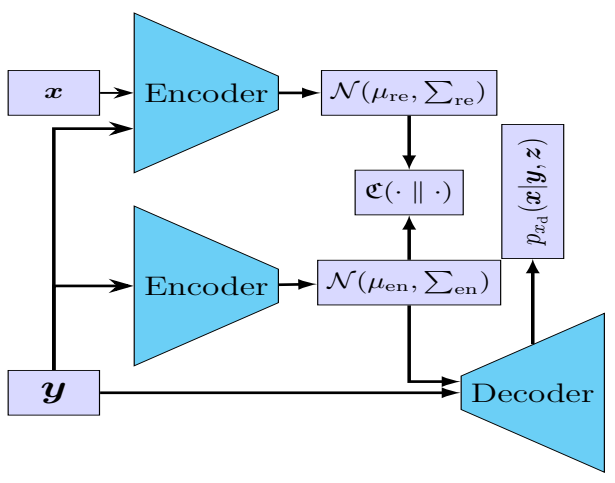

(a)

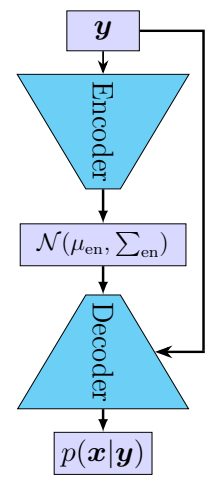

(b)
Fig. 2. The framework of the proposed CVAE: (a) during training; (b) during test.

\section{Simulation Results}

The proposed method is tested in the IEEE 39-bus system using PSS/E software. In the case study, the base contingency 
TABLE I

Calibration of the Composite Load Parameters.

\begin{tabular}{|c|c|c|c|c|c|c|c|c|c|c|c|}
\hline Param. & $\begin{array}{l}\text { True } \\
\text { Value }\end{array}$ & Estimated & Param. & $\begin{array}{c}\text { True } \\
\text { Value }\end{array}$ & Estimated & Param. & $\begin{array}{c}\text { True } \\
\text { Value }\end{array}$ & Estimated & Param. & $\begin{array}{c}\text { True } \\
\text { Value }\end{array}$ & Estimated \\
\hline \multicolumn{3}{|c|}{ Motor A } & $E_{\mathrm{trq}}$ & 2 & 1.95 & $N_{\mathrm{p} 2}$ & 3.2 & 3.22 & $T_{\mathrm{rf}}$ & 0.005 & 0.005 \\
\hline$T_{\mathrm{po}}$ & 0.095 & 0.094 & $L_{\mathrm{a}}$ & 0.059 & 0.62 & $N_{\mathrm{q} 1}$ & 2 & 2.1 & $K_{\mathrm{qv}}$ & 1 & 1 \\
\hline$T_{\mathrm{ppo}}$ & 0.002 & 0.002 & \multicolumn{3}{|c|}{ Motor C } & $N_{\mathrm{q} 2}$ & 2.5 & 2.3 & $T_{\mathrm{p}}$ & 0.025 & 0.022 \\
\hline$L_{\mathrm{p}}$ & 1.8 & 1.7 & $T_{\mathrm{po}}$ & 0.2 & 0.21 & $C M K P F$ & 1 & 0.92 & $T_{\mathrm{iq}}$ & 0.02 & 0.02 \\
\hline$L_{\mathrm{pp}}$ & 0.12 & 0.1 & $T_{\mathrm{ppo}}$ & 0.003 & 0.003 & $C M K Q F$ & -3.3 & -3.32 & $T_{\text {pord }}$ & 0.1 & 0.1 \\
\hline$L_{\mathrm{s}}$ & 0.04 & 0.41 & $L_{\mathrm{p}}$ & 1.8 & 1.82 & \multicolumn{3}{|c|}{ Static Load } & $K_{\mathrm{pg}}$ & 0.1 & 0.1 \\
\hline$R_{\mathrm{S}}$ & 0.104 & 0.102 & $L_{\mathrm{pp}}$ & 0.19 & 0.21 & $P_{1 \mathrm{c}}$ & 0.648 & 0.645 & $K_{\text {ig }}$ & 0.05 & 0.05 \\
\hline$H$ & 0.1 & 0.1 & $L_{\mathrm{s}}$ & 0.03 & 0.2 & $P_{2 \mathrm{c}}$ & 0.35 & 0.31 & $T_{\mathrm{g}}$ & 0.02 & 0.02 \\
\hline$E_{\mathrm{trq}}$ & 0.01 & 0.01 & $R_{\mathrm{S}}$ & 0.14 & 0.16 & $Q_{1 \mathrm{c}}$ & 1 & 0.8 & $T_{\mathrm{v}}$ & 0.2 & 0.21 \\
\hline$L_{\mathrm{a}}$ & 0.059 & 0.06 & $H$ & 0.1 & 0.1 & $Q_{2 \mathrm{c}}$ & 0.01 & 0.01 & $X_{\mathrm{e}}$ & 0.5 & 0.5 \\
\hline \multicolumn{3}{|c|}{ Motor B } & $E_{\text {trq }}$ & 2 & 2 & $P_{\text {freq }}$ & 0.001 & 0.001 & $L_{\text {fder }}$ & 0.75 & 0.76 \\
\hline$T_{\mathrm{po}}$ & 0.2 & 0.18 & $L_{\mathrm{s}}$ & 0.059 & 0.06 & $Q_{\text {freq }}$ & -1 & -0.95 & \multicolumn{3}{|c|}{ Load Fraction } \\
\hline$T_{\mathrm{ppo}}$ & 0.003 & 0.003 & \multicolumn{3}{|c|}{ Motor D } & \multicolumn{3}{|c|}{ Electronic load } & $F_{\mathrm{MA}}$ & 0.109 & 0.10 \\
\hline$L_{\mathrm{p}}$ & 1.8 & 1.75 & $K_{\mathrm{p} 1}$ & 0.1 & 0.1 & $F_{\text {rcel }}$ & 0.6 & 0.6 & $F_{\mathrm{MB}}$ & 0.114 & 0.11 \\
\hline$L_{\mathrm{pp}}$ & 0.19 & 0.18 & $K_{\mathrm{p} 2}$ & 12 & 11.85 & $V_{\mathrm{d} 1}$ & 0.5 & 0.52 & $F_{\mathrm{MC}}$ & 0.061 & 0.061 \\
\hline$L_{\mathrm{s}}$ & 0.03 & 0.03 & $K_{\mathrm{q} 1}$ & 6 & 6.1 & $V_{\mathrm{d} 2}$ & 0.8 & 0.76 & $F_{\mathrm{MD}}$ & 0.086 & 0.85 \\
\hline$R_{\mathrm{s}}$ & 0.14 & 0.135 & $K_{\mathrm{q} 2}$ & 11 & 11.1 & \multicolumn{3}{|c|}{ DER A } & $F_{\mathrm{el}}$ & 0.189 & 0.19 \\
\hline$H$ & 0.5 & 0.51 & $N_{\mathrm{p} 1}$ & 1 & 0.9 & $T_{\mathrm{rv}}$ & 0.008 & 0.007 & $P_{\text {fel }}$ & 1 & 1 \\
\hline
\end{tabular}

is chosen as a three-phase fault occurred at bus 6 . We consider a dynamic load with CMLDBLDGU2 model [14] located at bus 20 .

\section{A. Hyper-parameter Setting and Dataset}

The selection of appropriate hyper-parameters is often necessary for satisfactory performance in learning-based methods. In this paper, a grid search optimization method via scikitlearn [21] is used to find the network hyper-parameters, such as batch size, max-pool size, stride length, the size of the latent space, learning rate, and etc. For the fully connected layers, we use a dropout of 0.3. The max-pooling layers have a pool size of 50 and a stride length of 50. All the case studies are tested using the Python 3.8 and the deep learning is carried out using a neural networks API, PyTorch [22].

In order to achieve high accuracy, increasing the number of simulations is desirable; however, it will cause a greater computational burden. Multiple critical parameters are randomly chosen and we automatically generate dynamic generator data with respect to them. Based on our numerical experiments, 3000 simulations are sufficient to estimate 64 parameters of the composite load model. We choose $90 \%$ of the available data for training and cross-validation, and $10 \%$ for testing. For each simulation, the time horizon for getting the whole dynamic responses is set as $10 \mathrm{~s}$.

\section{B. Calibration of High Dimensional Case}

This case study demonstrates the performance of the proposed model in a higher-dimensional case with 64 parameters. We select the 64 parameters same as [5], [13]. The prior distributions of these parameters for training the model are assumed to have a $\mu_{\pi}\left(\alpha_{\mathrm{c}}\right)$ with $40 \%$ deviation from the true values and follow a uniform distribution as $\mathcal{U}\left(\boldsymbol{\mu}_{\pi}\left(\boldsymbol{\alpha}_{\mathrm{c}}\right)-\right.$ $\left.\boldsymbol{\mu}_{\pi}\left(\boldsymbol{\alpha}_{\mathrm{c}}\right) \cdot 40 \%, \boldsymbol{\mu}_{\pi}\left(\boldsymbol{\alpha}_{\mathrm{c}}\right)+\boldsymbol{\mu}_{\pi}\left(\boldsymbol{\alpha}_{\mathrm{c}}\right) \cdot 40 \%\right)$.

Table I shows the true and corresponding identified parameter values. As can be observed, the proposed approach can give us satisfactory results. The accuracy of the identification can be further confirmed in Fig. 3, where the estimated active power and reactive power of the line between bus 20 and 34 and voltage at bus 20 curves closely match the actual curves.

True model parameters are never known in real-world applications. Different events may result in different parameters being provided by the algorithm. The parameters can be estimated from one event, and the cross-validation process can be conducted using the other events. Here we assume measurements for two events are available. We consider a three-phase fault at bus 14 for the second event. Fig. 4 shows the results of the estimated and the measurements of the model with another event. It can be concluded that the identified parameters also yield good matches for different events.

\section{CONClusions And Future Works}

The paper presents a parameter identification approach for WECC composite load models. The identification of parameters is performed using a cutting-edge method based on deep learning. Our numerical experiments on IEEE 39-bus system demonstrate that the proposed approach can achieve promising results. Comparatively to data-hungry methods that require large sets of disturbances to calibrate, the proposed method only requires a set of reference dynamic responses. In our future work, we will develop a method to quantify 


\section{REFERENCES}
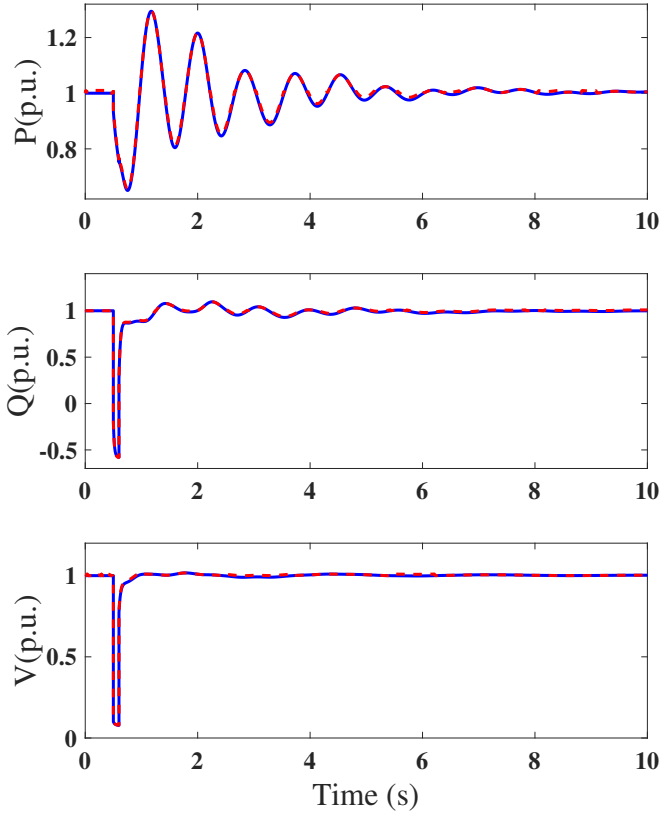

Fig. 3. The real power, the reactive power, and the voltage curves for the measurements and identified parameters.
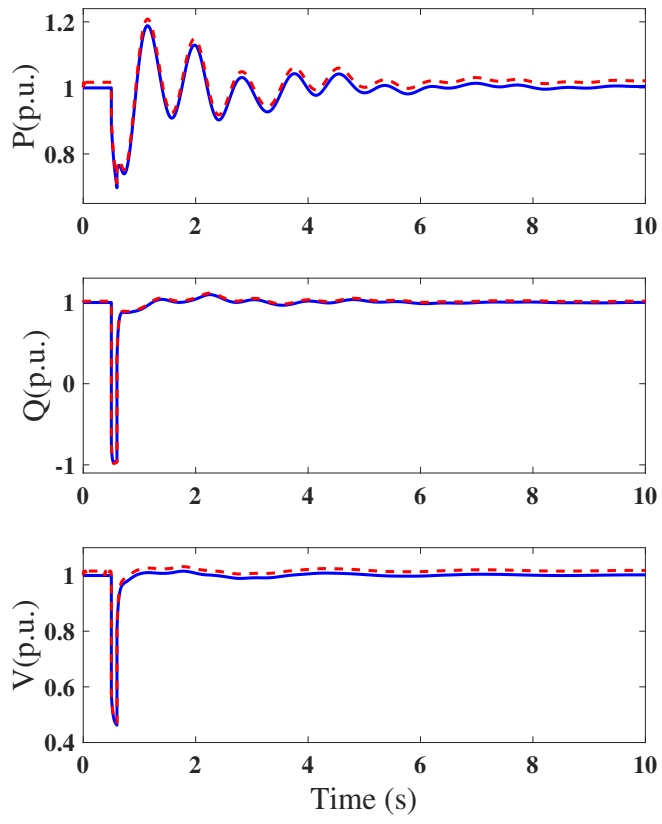

Fig. 4. The real power, the reactive power, and the voltage curves for the measurements and identified parameters for the second event.

the parameter-output behavior of composite load models from the perspective of nonlinear systems and identify critical parameters.
[1] A. Rabiee, S. M. Mohseni-Bonab, M. Parniani, and I. Kamwa, "Optimal cost of voltage security control using voltage dependent load models in presence of demand response," IEEE Trans. Smart Grid, vol. 10, no. 3, pp. 2383-2395, 2018.

[2] M. Tasdighi, H. Ghasemi, and A. Rahimi-Kian, "Residential microgrid scheduling based on smart meters data and temperature dependent thermal load modeling," IEEE Trans. Smart Grid, vol. 5, no. 1, pp. 349-357, 2013.

[3] Q. Huang and V. Vittal, "Application of electromagnetic transienttransient stability hybrid simulation to fidvr study," IEEE Trans. Power Syst., vol. 31, no. 4, pp. 2634-2646, 2015.

[4] J. Zhao, Z. Wang, and J. Wang, "Robust time-varying load modeling for conservation voltage reduction assessment," IEEE Trans. Smart Grid, vol. 9, no. 4, pp. 3304-3312, 2016.

[5] F. Bu, Z. Ma, Y. Yuan, and Z. Wang, "Wecc composite load model parameter identification using evolutionary deep reinforcement learning," IEEE Trans. Smart Grid, vol. 11, no. 6, pp. 5407-5417, 2020.

[6] S. R. Khazeiynasab and J. Qi, "Generator parameter calibration by adaptive approximate bayesian computation with sequential monte carlo sampler," IEEE Trans. Smart Grid, May 2021.

[7] H. Haggi, W. Sun, J. M. Fenton, and P. Brooker, "Risk-averse cooperative operation of $\mathrm{pv}$ and hydrogen systems in active distribution networks," IEEE Systems Journal, Sept. 2021.

[8] M. Cui, M. Khodayar, C. Chen, X. Wang, Y. Zhang, and M. E. Khodayar, "Deep learning-based time-varying parameter identification for systemwide load modeling," IEEE Trans. Smart Grid, vol. 10, no. 6, pp. 61026114, Jan. 2019.

[9] M. Khodayar and J. Wang, "Probabilistic time-varying parameter identification for load modeling: A deep generative approach," IEEE Trans Industr Inform ., vol. 17, no. 3, pp. 1625-1636, 2020.

[10] J. Xie, Z. Ma, K. Dehghanpour, Z. Wang, Y. Wang, R. Diao, and D. Shi, "Imitation and transfer q-learning-based parameter identification for composite load modeling," IEEE Trans. Smart Grid, 2020.

[11] K. Zhang, H. Zhu, and S. Guo, "Dependency analysis and improved parameter estimation for dynamic composite load modeling," IEEE Trans. Power Syst., vol. 32, no. 4, pp. 3287-3297, 2016.

[12] X. Wang, Y. Wang, D. Shi, J. Wang, and Z. Wang, "Two-stage wecc composite load modeling: A double deep q-learning networks approach," IEEE Trans. Smart Grid, vol. 11, no. 5, pp. 4331-4344, Apr. 2020.

[13] Z. Ma, B. Cui, Z. Wang, and D. Zhao, "Parameter reduction of composite load model using active subspace method," IEEE Trans. Power Syst., 2021.

[14] P. Siemens, "PSS®E 35.2.1 model library," PSS/E Manual, 2021.

[15] D. P. Kingma and M. Welling, "Auto-encoding variational bayes," arXiv preprint arXiv:1312.6114, Dec. 2013.

[16] O. Ivanov, M. Figurnov, and D. Vetrov, "Variational autoencoder with arbitrary conditioning," arXiv preprint arXiv:1806.02382, Jun. 2018.

[17] K. Sohn, H. Lee, and X. Yan, "Learning structured output representation using deep conditional generative models," NeurIPS, vol. 28, pp. 34833491, 2015.

[18] H. Gabbard, C. Messenger, I. S. Heng, F. Tonolini, and R. MurraySmith, "Bayesian parameter estimation using conditional variational autoencoders for gravitational-wave astronomy," arXiv preprint arXiv:1909.06296, 2019.

[19] B. Ivanovic, K. Leung, E. Schmerling, and M. Pavone, "Multimodal deep generative models for trajectory prediction: A conditional variational autoencoder approach," IEEE Robot. Autom. Lett, vol. 6, no. 2, pp. 295302, Dec. 2020.

[20] S. R. Khazeiynasab, J. Zhao, I. Batarseh, and B. Tan, "Power plant model parameter calibration using conditional variational autoencoder," IEEE Transactions on Power Systems, 2021.

[21] F. Pedregosa, G. Varoquaux, A. Gramfort, V. Michel, B. Thirion, O. Grisel, M. Blondel, P. Prettenhofer, R. Weiss, V. Dubourg et al., "Scikit-learn: Machine learning in python," J Mach Learn Res, vol. 12, pp. 2825-2830, Nov. 2011.

[22] A. Paszke, S. Gross, F. Massa, A. Lerer, J. Bradbury, G. Chanan, T. Killeen, Z. Lin, N. Gimelshein, L. Antiga et al., "Pytorch: An imperative style, high-performance deep learning library," in NeurIPS. Curran Associates, Inc., 2019, pp. 8024-8035. 